Gut, 1986, 27, 1018-1024

\title{
Physiologic study of the terminal digestive tract in chronic painful constipation
}

\author{
PATRICK MEUNIER \\ From the Manometric Investigation Laboratory, INSERM U 45, Faculté de Médecine Lyon-Nord, Lyon, \\ France
}

SUMMARY A manometric study of the sigmoid colon and of the anorectum was undertaken in 65 chronically constipated patients complaining of abdominal pain, and in a control group of 23 healthy volunteers. Rectal compliance was tested in both groups. The sigmoid motility study allowed for the segregation of the constipated patients into three groups: hypokinesia (12 cases), normokinesia (34 cases), hyperkinesia (19 cases). Rectal manometry showed anal hypertony in 24 patients, impaired rectal conscious sensitivity in 12 subjects, and normal functions in the remaining cases. The rectal compliance study disclosed a decreased compliance in 15 cases and increased compliance in 13 other patients. In 12 cases disordered sigmoid motility was the only abnormality; in 10 cases only a rectoanal abnormality was found. Most of the patients (52\%) exhibited miscellaneous disorders. In contrast, all parameters were normal in nine subjects. No consistent pattern of motility disorders was thus demonstrated in this clinically homogeneous group of patients with chronic, painful, constipation.

Since the classical study by Connell ${ }^{1}$, the major motor disorder, in chronic constipation, has been considered to be an over segmentation of the sigmoid colon that provides a pressure barrier on the transit of stools toward the rectum. Although this concept has often been confirmed, ${ }^{2-7}$ whatever the underlying disorder, ${ }^{7}$ the hypothesis of a single mechanism of constipation might well be disputed. Indeed a simple digital examination proves the high incidence of stool retention within the rectum in a number of constipated patients. Moreover, the radiopaque pellet study by Martelli $e t \mathrm{al}^{8}$ shows that constipated patients can be segregated into three main classes, namely whole colon inertia, hindgut dysfunction, and outlet obstruction. Further, in a previous study of constipated patients, we observed that motility of the sigmoid colon was most often normal. ${ }^{9}$ Finally, various studies ${ }^{10-16}$ have shown a high incidence of anorectal disorders in this clinical condition.

Thus, it clearly appears that chronic constipation does represent a complex pathophysiological situation. The present work was designed to further

Address for correspondence: Dr Patrick Meunier, INSERM U 45, Pavillon Hbis, Hôpital Edouard Herriot, 69374 Lyon Cédex 08, France.

Received for publication 3 January 1986. characterise the colorectoanal motility disorders involved in chronic constipation in adults. Inclusion was restricted to patients complaining of abdominal pain and from constipation. Manometric profiles of sigmoid colon anorectum, and rectal compliance were studied for comparison in this clinical group and in healthy volunteers.

\section{Methods}

\section{SUBJECTS AND PATIENTS}

The complete investigation was carried out on 23 healthy volunteers (control subjects), and on 65 chronically constipated patients. In the later group all medication, if any, was interrupted eight days before the procedure. Regular users of psychotropic drugs were excluded from this study.

The control subjects, six women and 17 men aged 19-26 years (median 22), were healthy medical students, with no history of gastrointestinal disease, and with normal bowel habits. According to previous studies, ${ }^{1718}$ normality in bowel habits was defined as the passage of at least three normal stools per week and of less than three stools per day. Written informed consent was obtained from each subject.

The constipated patients were 19 men and 46 
women, aged 17-65 years (median 39). Inclusion was based on the following criteria: onset of symptoms during childhood, recurrent attacks of abdominal pain (at least 10 per year), normal barium enema and coloscopy, normal serum electrolytes, long abuse of laxatives, recent failure of bran or lactulose therapy, and recent worsening of symptoms. Without treatment, stool frequency in these patients was less than one every three days, but in all cases, except during the washout period of eight days preceding the investigation, the frequency and consistency of stools actually depended upon the use of laxative or enema. Gastrointestinal, metabolic, neurologic, and drug induced causes for the alteration in bowel habits, were thoroughly searched. Primary constipation with recurrent abdominal pain was the only complaint in these patients.

The following procedure was followed during a single test period by both controls and constipated patients, after an 18-20 hours fast. A cleansing enema ( $\mathrm{Normacol}^{\circledR}$ ) was administered four to six hours before each study. During the complete investigation, the patients rested in the left supine position; they were allowed to read, but smoking and sleeping were not allowed. The test consisted of the three successive stages: (1) sigmoid motility study, (2) rectoanal manometric study, (3) rectal compliance study. To avoid observer divergences, the whole investigation, including interpretation of recordings, and calculations were done by the author only. As the methods used have already been described, ${ }^{1116}$ only the essential parts will be reported here, except where modifications were introduced. Pressure determinations are expressed in kiloPascal $(\mathrm{kPa}), 1 \mathrm{kPa}=9.81 \mathrm{~cm}$ water. Calibration of the recording device was done using a water column.

\section{SIGMOID MOTILITY STUDY}

A double lumen catheter with side openings spaced at $10 \mathrm{~cm}$ intervals was introduced into the sigmoid colon through a rigid rectoscope, ensuring that the two openings were at approximately 25 and $15 \mathrm{~cm}$ from the anal margin. The total length of the catheter was $40 \mathrm{~cm}$, each lumen had an internal diameter of $1.5 \mathrm{~mm}$, and the side openings had a diameter of $1 \mathrm{~mm}$. The two lumens were perfused with water and connected to a galvinometric recorder via pressure transducers and DC amplifiers. Motor activity was recorded for 30 minutes while the subjects were fasted (basal period). A meal was then eaten in 10 minutes at most; the energy value of this meal varied between 3750 and $4600 \mathrm{~kJ}$ (approximately 900 to $1100 \mathrm{kCal}$ ). The recording of sigmoid motor activity was continued for $30 \mathrm{~min}$ after the meal. Only those recordings obtained at $25 \mathrm{~cm}$ (in the sigmoid) were analysed, recordings at $15 \mathrm{~cm}$ (in the rectum) were used to accurately detect artefacts. The motility index (percentage of activity $x$ mean amplitude of waves $\times 10$ ) was calculated for both periods of recording. The sigmoid motility study provided two parameters: the basal sigmoid motility index, and the postprandial sigmoid motility index.

\section{RECTOANAL MANOMETRIC STUDY}

For the second step of the investigation a tandem system of two side-opening perfused catheters was used. The openings were inserted at approximately $8 \mathrm{~cm}$ (in the rectum), and $2.5 \mathrm{~cm}$ (in the upper anal canal), above the anal margin. In addition, a tube on which a latex balloon was mounted, was inserted into the rectal ampula. This balloon was used to distend the rectal wall with air, thus allowing the study of the rectoanal inhibitory reflex - that is, a transient anal relaxation after a brief rectal distension, and of the rectorectal reflex - that is, rectal contraction in response to rectal distension. The thresholds for these two reflexes were noted and expressed as $\mathrm{ml}$ : the rectoanal inhibitory reflex threshold was the distension volume at which a minimal anal relaxation of $1 \mathrm{kPa}$ occurred, the rectorectal reflex threshold the distension volume at which a minimal rectal contraction of $1 \mathrm{kPa}$ occurred.

The rectal pressure in absence of any distending stimulus, that is the rectal pressure at rest, was noted and expressed in $\mathrm{kPa}$.

The conscious rectal sensitivity threshold - that is the distension volume for which an initial transient sensation occurred was determined, by questioning the patient, and was expressed in $\mathrm{ml}$.

Subtracting the rectal pressure from the maximal anal pressure gave a rectoanal pressure profile which allowed for determination of the maximal anal resting closure pressure. This was expressed in $\mathrm{kPa}$ and was obtained using a perfused side opening catheter, pulled at $20 \mathrm{~cm} / \mathrm{min}$ from the rectum to the anal verge.

\section{RECTAL COMPLIANCE STUDY}

At this stage of the investigation, a pressure volume curve of the rectum - that is, a rectometrogram, was recorded, using a double lumen catheter on which a distending balloon was mounted. The balloon was a simple condom cut so that its total length was $12 \mathrm{~cm}$, and the distal part of the balloon was placed at 4-5 $\mathrm{cm}$ from the anal margin. One lumen of the catheter was used to stepwise inflate the rectal ampula with air volumes of $50 \mathrm{ml}$. The second lumen was used to measure the pressure in the balloon. Between each air injection, a latency time $(30-60 \mathrm{~s})$ was ensured to allow the rectal wall for adapting to the new volume, 
so that the subsequent injection of air was made only after obtaining a plateau of rectal pressure. Inflation was immediately stopped when the patient experienced a painful sensation. At this stage, the maximal rectal tolerable volume (expressed in $\mathrm{ml}$ ) was attained; the corresponding pressure was the maximal rectal tolerable pressure (expressed in $\mathrm{kPa}$ ). The ratio of the maximal rectal volume to the maximal rectal pressure was defined as the rectal compliance and expressed in $\mathrm{ml} / \mathrm{kPa}$.

\section{STATISTICAL ANALYSIS}

Results are expressed as median, range, and interquartile range. Data were analysed using nonparametric tests for ranked values. ${ }^{19}$ Statistical comparisons were made using the Mann-Whitney $U$ test for two independent samples, the Wilcoxon's test for paired data and the $\chi^{2}$ test for $\mathrm{k}$ independent samples.

\section{Results}

To test the homogeneity of the results between male and female control subjects, a statistical analysis was carried out on the ranked values of the eight parameters of this study. The $U$ test disclosed no sex linked difference of the sigmoid and rectoanal parameters. Consequently, the results observed in controls and constipated patients were analysed as a whole within each group, and are presented in Table 1. Except for the basal and postprandial sigmoid motility index, and the maximal rectal compliance, the parameters studied in the two groups were not statistically different. The median values for each parameter were very similar in controls and pa- tients, but in most cases the distribution of individual values widely varied within each group. A number of constipated patients showed individual values outside the control ranges. A detailed analysis of the results was thus undertaken, assuming that the distribution of values in the control population defined the normal range of each parameter. The abnormal values found for the parameter of this study are presented in Table 2 .

In the sigmoid motility study, the postprandial motility index was better able to reveal motor abnormalities than the basal motility index. Indeed the basal sigmoid motility index isolated only nine patients with increased sigmoid motility (hyperkinesia), and none with decreased sigmoid motility (hypokinesia); while with the postprandial sigmoid motility index 19 cases of hyperkinesia, and 12 cases of hypokinesia were identified. On the other hand, the Wilcoxon's test proved that both in controls and patients, the postprandial motility index was significantly higher than the basal motility index $(p>0.001)$. Therefore, for the purpose of this study, the postprandial motility index was considered to afford better information than the basal motility index, confirming our previous observations. ${ }^{9}$ The rectoanal inhibitory reflex was present in each constipated subject, which ruled out the possibility of undetected Hirschsprung's disease in this series. ${ }^{20-22}$ The distributions of individual thresholds for this reflex, however, were similar in control and constipated subjects. Similarly values in the two groups for the rectal pressure at rest and the rectorectal reflex threshold were not different. Therefore, the rectoanal inhibitory reflex threshold, the rectal pressure at rest, and the rectorectal reflex threshold

Table 1 Medican interquartile range, and range of the sigmoid are rectoanal parameters in controls and painfully constipated patients.

\begin{tabular}{|c|c|c|c|c|c|c|c|}
\hline & \multicolumn{3}{|c|}{ Control subjects } & \multicolumn{4}{|c|}{ Constipated subjects } \\
\hline & \multicolumn{3}{|c|}{ Interquartile } & \multicolumn{4}{|c|}{ Interquartile } \\
\hline & Median & Range & Range & Median & Range & Range & $P$ \\
\hline BSMI & 128 & $64-176$ & $0-231$ & 91 & $37-165$ & $0-891$ & 0.001 \\
\hline PPSMI & 528 & $325-816$ & $240-946$ & 540 & $360-1110$ & $54-2184$ & 0.05 \\
\hline RAIRT (ml) & 20 & $10-30$ & $5-40$ & 15 & $10-30$ & $5-40$ & NS \\
\hline RPR (KPa) & $1 \cdot 0$ & $0 \cdot 8-1 \cdot 2$ & $0 \cdot 6-1.7$ & $1 \cdot 1$ & $0 \cdot 8-1 \cdot 3$ & $0 \cdot 6-1 \cdot 8$ & NS \\
\hline RRRT (ml) & 25 & $10-30$ & $5-40$ & 20 & $10-30$ & $5-90$ & NS \\
\hline CRST (ml) & 15 & $5-20$ & $5-35$ & 20 & $10-30$ & $5-220$ & NS \\
\hline MARPC (KPa) & 8 & $7 \cdot 5-9 \cdot 5$ & $6-11$ & $9 \cdot 5$ & $7-13 \cdot 5$ & $5 \cdot 5-19$ & NS \\
\hline MRC (mi/KPa) & 43 & $34-54$ & $28-63$ & 46 & $29 \cdot 5-65$ & $11-343$ & 0.05 \\
\hline
\end{tabular}

Except for the BSMI, the PSSMI, the MRTP and the MRC these parameters were not significantly different between the two populations (see last column of Tables).

BSMI = basal sigmoid index; PPSMI = postprandial sigmoid motility index; RAIRT =rectoanal inhibitory reflex threshold; $R P R=$ rectal pressure at rest; $\mathrm{RRRT}=$ rectorectal reflex threshold; $\mathrm{CRST}=$ conscious rectal sensitivity threshold; MARPC=maximal anal resting closure pressure; $\mathrm{MRC}=$ maximal rectal compliance. 
Table 2 Number, percentage, median and range of values found outside the normal range in the constipated patients.

\begin{tabular}{llcccc}
\hline & & Number & Percentage & Median & Range \\
\hline BSMI & Higher & 9 & 14 & 384 & $240-891$ \\
PPSMI & Lower & 12 & 18 & 115 & $54-192$ \\
RRT & Higher & 19 & 5 & 1676 & $984-2184$ \\
CRST & Higher & 3 & 18 & 60 & $60-70$ \\
MARCP & Higher & 12 & 37 & 60 & $40-220$ \\
MRC & Higher & 24 & 23 & 14 & $11-26$ \\
& Lower & 15 & 20 & 126 & $65-343$ \\
\hline
\end{tabular}

It must be noted that for the RAIRT none of the patients were out of the normal range; for the RPR only one patient was outside of the normal range with a value of $1.8 \mathrm{KPa}$; for the MARCP only two patients were under normal range ( $5.5 \mathrm{KPa}$ in each case). All these cases are not presented in this table. (For definition of abbreviations see Table 1.)

were not deemed to afford useful data in this study, so that the postprandial sigmoid motility index, the conscious rectal sensitivity threshold, the maximal anal pressure at rest, and the maximal rectal compliance only proved adequate to show sigmoid and rectoanal abnormalities in these constipated patients with abdominal pain.

In 12 patients $(18 \%)$, an abnormal postprandial sigmoid motility was the only disorder, including seven cases with hyperkinesia and five cases with hypokinesia. Anal hypertonia (high anal pressure) was the only abnormality in five other patients $(8 \%)$. Five patients showed only an abnormal maximal rectal compliance, increased in one case and decreased in the four other subjects. In two patients, the only disorder was a high rectal sensitivity threshold ( $40 \mathrm{ml}$ in both cases); these thresholds were, however, close to the upper limit of normal, and were not considered as conclusive evidence for disordered rectoanal motility.

In the 34 other patients (52\% of the total group), there was an association of either sigmoid hypokinesia or hyperkinesia with various rectoanal abnormalities. Using $\chi^{2}$ tests we attempted to find a correlation between the sigmoid motility disorder (hyperkinesia or hypokinesia) and the rectoanal parameters, but no significant relationship was found.

In 27 patients (42\% of the total group) a normal postprandial motility index (normokinesia) was associated with abnormal rectoanal parameters. No typical rectoanal disorder emerged in this subgroup, each possible rectoanal abnormality being observed in at least some patients. In addition most patients showed multiparametric alterations of rectoanal motility. Statistical analysis with $\chi^{2}$ tests did not reveal any correlation between these rectoanal anomalies, although an association of high anal pressure with high rectal compliance was recorded in 11 patients ( $44 \%$ of this clinical subgroup).
In this group of 65 constipated patients with pain, seven individuals $(11 \%)$ were found to be fully normal. If the two patients with a slight increase of rectal sensitivity threshold were included, the percentage of patients with normal profiles would reach as high as $14 \%$ of the total group.

\section{Discussion}

In this selected series of patients with painful constipation no typical pattern of abnormality was recorded. This observation is fairly consistent with the complexity of the motor dysfunctions reputed to be involved in adult constipation. ${ }^{202324}$ On the other hand the present results gathered in an homogenous group of patients are disappointing and have to be discussed with respect to methodology and group selection. The first point is the fact that control subjects and patients were not, for practical reasons, matched for age and sex. The present control population adequately consisted of young, healthy subjects, responding to the criteria usually adopted to define a physiological range of values. No difference was found between women and men for the various parameters. Furthermore Wyman et al. ${ }^{25}$ showed that the colonic functions in healthy subjects do not vary significantly with age and sex. Consequently, the bias resulting from studying two groups not matched for age and sex probably played little role in the present comparison.

Secondly, the search for abnormalities of sigmoid motility through a sigmoid manometric study is questionable, as we previously reported normal sigmoid motility indices in most constipated patients. ${ }^{9}$ Also the wide range of the motility index in healthy subjects ${ }^{926}$ and the large day to day individual variations of both colonic motility ${ }^{27}$ and functions ${ }^{25}$ are obvious limitations of sigmoid manometric studies for clinical purposes. Nevertheless, we show here, as previously, ${ }^{9}$ that the postprandial 
motility index allows for an easy segregation of constipated patients into three groups (normo, hypo, and hyperkinesia). The constipated subjects with normal sigmoid motility are possibly those showing normal colonic transit times as described in some pellets studies $;{ }^{82}$ hypokinetic patients may suffer from constipation with colonic inertia; ${ }^{8}$ patients with hyperkinesia probably belong to those cases of irritable bowel syndrome where sigmoid oversegmentation can be accurately related to pain. ${ }^{29}{ }^{30}$ Loening-Baucke and Anuras ${ }^{31}$ recently claimed that there was no sigmoid motility increase after a meal. This clearly raised the question of whether it is legitimate or not to use postprandial colonic motility studies in clinical research. In fact, the results of these authors are in complete disagreement with most previous works. ${ }^{34932-34}$ They used a flexible probe, introduced blindly in the lower bowel. Daily experience shows that accurate positioning of the catheter in the sigmoid requires endoscopy, so that the above tests may have concerned the rectum, rather than the sigmoid colon. Thompson ${ }^{35}$ recently stated that the colon moves in ways too complicated to be assessed by the 'primitive' methods presently available. From the present and previous results ${ }^{9}$ we propose that postprandial manometry of the sigmoid is indeed a useful investigation tool in severe constipation. The fact must be emphasised, however, that sigmoid motility recordings do not, by any means, allow for extrapolating the results to other parts of the colon. ${ }^{36}$

The value of the rectoanal investigation here undertaken, is less controversial. ${ }^{2022} 23$ To check the presence of a rectoanal inhibitory reflex is mandatory in patients with severe constipation, ${ }^{22}$ especially in the present group because the onset of constipation occurred during childhood.

In the present study a minority of patients with a decrease of conscious rectal sensitivity, a fact at clear variance with what has been described both in constipated children, ${ }^{113738}$ and in the elderly. ${ }^{24} 39$ Although constipated patients frequently report on the lack of defecatory urges, ${ }^{24}$ rectal sensitivity studies have been rarely done in adults. In the works of Ihre, ${ }^{40}$ and of Baldi $e t$ al $^{12}$ an impaired rectal sensation was the only rectoanal abnormality observed in constipated adults. On the contrary, only $18 \%$ of our constipated patients showed an improved rectal sensitivity - that is, a higher conscious rectal sensitivity threshold. This discrepancy is probably related to the characteristics of the clinical group here tested, namely patients with painful constipation, as patients with abdominal pain are known to be very sensitive to intestinal tract distension. ${ }^{4142}$ Clearly, the study of rectal sensi- tivity is another important tool in the investigation of defecation disorders, provided the clinical status of the patients is evaluated as precisely as possible.

Resting anal closure was increased in $37 \%$ of the patients examined in the course of this study, a figure very close to the one we recently reported in children. ${ }^{16}$ Similar results have been obtained in other studies in children, ${ }^{11}{ }^{14}$ but only incidentally in adults. ${ }^{8}$ Probably anal hypertonia has rarely been reported in constipated adults because anal tone was not systematically measured in studies on constipation in adults.

In $43 \%$ of the constipated patients, the rectometrogram obtained showed abnormalities of the pressure-volume relationship. Both high and low values of maximal compliance were observed. Low rectal compliance was recorded in $23 \%$ of the cases, suggesting that these patients had an irritable lower bowel because decreased rectal distension thresholds have consistently been reported in this clinical condition. ${ }^{43}$ Though they complained of painful constipation, $20 \%$ of the patients showed an altered responsiveness of the rectal wall to distension (high rectal compliance). This suggests that the pain experienced by such patients actually originated from other structures than the pelvic bowel itself. Compliance studies probably offer a more accurate way of assessing rectal wall abnormalities than sensitivity thresholds do, ${ }^{16} 44{ }^{45}$ because the thresholds of distension induced sensation in the gut largely depend on the characteristics of the distending stimulus. ${ }^{46}$

A substantial proportion of patients were fully normal according to the present investigation, and it is tempting to claim that these subjects had a purely psychogenic disorder - that is, the failure to recognise or to respond to defecatory urges. This study, however, does not allow for any firm conclusion according to which a non-negligible percentage of constipated adults would have strictly normal lower bowel functions, because other investigation tools such as peptides biochemistry, histology, psychophysic, and electrophysiology, would probably reveal some discrete abnormalities outside the scope of manometry.

The present work shows the complexity of the motor disorders involved in adult patients with painful constipation, in spite of the fairly stereotyped clinical picture of this clinical group. The scatter of the results may imply that a number of mechanisms probably operate in various combinations for different subtypes of constipation, and that these subtypes cannot be completely identified with the classical methods here used. It is likely that both an extension of the manometric study to the proximal colon, and the repetition of the present 
tests during an attack of abdominal pain would lead to improved segregation of these patients. It remains that the individual manometric profile may help to adjust the therapeutic follow up by identifying the variable disorders that underlie a common clinical history.

This work was supported with grants from la Faculté de Biologie Humaine, Université Lyon I et la Mission de la Recherche, Ministère de l'Education Nationale, Paris. The material support of INSERM 45 is acknowledged. The author is grateful to Professor Chayvialle who provided much helpful advice.

\section{References}

1 Connell AM. The motility of the pelvic colon. II. Paradoxical motility in constipation and diarrhoea. Gut 1962; 3: 342-8.

2 Wangel AG, Deller DJ. Intestinal motility in man. III. Mechanisms of constipation and diarrhoea with particular reference to the irritable colon syndrome. Gastroenterology 1965; 11: 577-80.

3 Waller SL, Misiewicz JJ, Kiley N. Effect of eating on motility of the pelvic colon in constipation and diarrhoea. Gut 1972; 13: 805-11.

4 Misiewicz JJ. Colonic motility. Gut 1975; 16: 311-4.

5 Kirwan WO, Smith AN. Colonic propulsion in diverticular disease, idiopathic constipation, and the irritable bowel syndrome. Scand J Gastroenterol 1977; 12: 331-5.

6 Frieri G, Parisi F, Corazziari E, Caprilli R. Colonic electromyography in chronic constipation. Gastroenterology 1983; 84: 737-40.

7 Chowdhury AR, Dinoso VP, Lorber SH. Characterization of a hyperactive segment at the rectosigmoid junction. Gastroenterology 1976; 71: 584-8.

8 Martelli H, Devroede G, Arhan P, Duguay C. Mechanisms of idiopathic constipation: outlet obstruction. Gastroenterology 1978; 75: 623-31.

9 Meunier P, Rochas A, Lambert R. Motor activity of the sigmoid colon in chronic constipation: comparative study with normal subjects. Gut 1979; 20: 1095-101.

10 Tobon F, Schuster MM. Megacolon: special diagnostic and therapeutic features. Johns Hopkins Med J 1974; 135: 91-105.

11 Meunier P, Marechal JM, Jaubert de Beaujeu M. Rectoanal pressures and rectal sensitivity studies in chronic childhood constipation. Gastroenterology 1979; 77: 330-6.

12 Baldi F, Ferrarini P, Corinaldesi R, et al. Function of the internal anal sphincter and rectal sensitivity in idiopathic constipation. Digestion 1982; 24: 14-22.

13 Loening-Baucke VA, Younosazi MK. Abnormal and sphincter response in chronically constipated children. J Pediatr 1982; 100: 213-8.

14 Arhan P, Devroede G, Jehannin B, et al. Idiopathic disorders of fecal continence in children. Pediatrics 1983; 71: 774-9.

15 Lanfranchi GA, Bazzochi G, Brignola C, et al.
Different patterns of anorectal motility and gastrointestinal transit time in painful and not painful constipation. In: Roman C, ed Gastrointestinal motility. Lancaster: MTP Press, 1984: 476-82.

16 Meunier P, Louis D, Jaubert de Beaujeu M. Physiologic investigation of primary constipation in children: comparison with the barium enema study. Gastroenterology 1984; 87: 1351-57.

17 Martelli H, Devroede G, Arhan P, Duguay C, Dornic C, Faverdin C. Some parameters of large bowel motility in normal man. Gastroenterology 1978; 75: 612-8.

18 Drossman DA, Sandler RS, McKee D, et al. Bowel patterns among subjects not seeking health care. Gastroenterology 1982; 83: 529-34.

19 Siegel S. Nonparametric statistics for the behavioral sciences. Tokyo: McGraw-Hill Kogakusha Ltd, 1956.

20 Schuster MM. Megacolon in adults. In: Sleisenger MH, Fordtran JS, eds. Gastrointestinal diseases, 2 nd ed. Vol. II. Philadelphia: W. B. Saunders, 1978: 1812-24.

21 Meunier P, Marechal JM, Mollard P. Accuracy of the manometric diagnosis of Hirschsprung's disease. $J$ Pediatr Surg 1978; 13: 411-5.

22 Poisson J, Devroede G. Severe chronic constipation as a surgical problem. Surg Clin North Am 1983; 63: 193-217.

23 Devroede G. Constipation: mechanisms and management. In: Sleisenger MH, Fordtran JS, eds. Gastrointestinal diseases. 2nd ed. Vol I, Philadelphia: W B Saunders, 1978: 368-86.

24 Thompson WG. The irritable gut. Baltimore: University Park Press, 1979.

25 Wyman JB, Heaton KW, Manning AP, et al. Variability of colonic function in healthy subjects. Gut 1978; 19: $146-50$

26 Weinrich J, Andersen D. Intraluminal pressure in the sigmoid colon. I. Method and results in normal subjects. Scand J Gastroenterol 1976; 11: 577-80.

27 Dinoso VP, Murthy SNS, Goldstein J, Rosner B. Basal motor activity of the distal colon: a reappraisal. Gastroenterology 1983; 85: 637-42.

28 Hinton JM, Lennard-Jones JE. Constipation: definition and classification. Postgrad Med J 1968; 44: 720-3.

29 Holdstock DJ, Misiewicz JJ, Walker SL. Observations on the mechanism of abdominal pain. Gut 1969; 10: 19-31.

30 Connell AM, Jones FA, Rowlands EN. Motility of the pelvic colon. IV. Abdominal pain associated with colonic hypermotility after meals. Gut 1965; 6: 105-12.

31 Loening-Baucke V, Anuras S. Effects of a meal on the motility of the sigmoid colon and rectum in healthy adults. Am J Gastroenterol 1983; 78: 393-7.

32 Ritche JA. Colonic motor activity and bowel function. Part II. Distribution and incidence of motor activity at rest and after food and carbachol. Gut 1968; 9: 502-11.

33 Snape WJ, Matarazzo SA, Cohen S. Effect of eating and gastrointestinal hormones on human colonic myoelectrical and motor activity. Gastroenterology 1978; 75: 373-8.

34 Schang JC, Devroede G. Fasting and postprandial myoelectric spiking activity in the human sigmoid colon. Gastroenterology 1983; 85: 1048-53. 
35 Thompson WG. The irritable bowel. Gut 1984; 25: 305-20.

36 Kerlin P, Zinsmeister A, Phillips S, Motor response to food of the ileum, proximal colon, and distal colon of healthy humans. Gastroenterology 1983; 84: 762-70.

37 Callaghan RP, Nixon HH. Megarectum: physiological observations. Arch Dis Child 1964; 39: 153-7.

38 Meunier P, Mollard P, Marechal JM. Physiopathology of megarectum; the association of megarectum with encopresis. Gut 1976; 17: 224-7.

39 Newman HF, Freeman J. Physiologic factors affecting defecatory sensation: relation to aging. J Am Geriat Soc 1974; 12: 553-4.

40 Ihre $T$. Studies on anal function in continent and incontinent patients. Scand J Gastroenterol 1974; 9 suppl 25: 1-64.

41 Ritchie J. Pain from distension of the pelvic colon by inflating a balloon in the irritable bowel syndrome. Gut 1973; 6: 105-12.

42 Swarbrick ET, Hegarty JE, Bat L, Williams CB, Dawson AM. Site of pain from the irritable bowel. Lancet 1980; 2: 443-6.

43 Mitra R, Chura C, Rajendra JR, Schuster MM. Abnormal response to rectal distension in irritable bowel syndrome. [Abstract] Gastroenterology 1974; 66: A-116/770.

44 Arhan P, Devroede G, Danis K, et al. Viscoelastic properties of the rectal wall in Hirschsprung's disease. $J$ Clin Invest 1978; 62: 82-7.

45 Devroede G, Vobecky S, Masse S, et al. Ischemic fecal incontinence and rectal angina. Gastroenterology 1982; 83: $970-80$.

46 Lipkin M, Sleisenger MH. Studies of visceral pain: measurements of stimulus intensity and duration associated with the onset of pain in esophagus, ileum, and colon. J Clin Invest 1958; 37: 28-34. 